Kleine Wasserkraftwerke im Spannungsfeld zwischen Klima- und Gewässerschutz

\section{Zielkonflikte in der Umweltpolitik}

\author{
Umweltpolitik ist nicht mehr nur durch Konflikte mit anderen Politikfeldern, \\ sondern auch durch Zielkonflikte innerhalb des eigenen Bereiches gekennzeich- \\ net. So untersuchte das lÖW im Auftrag des Umweltbundesamtes die Frage, ob \\ kleine Wasserkraftwerke aus umweltpolitischer Sicht positiv oder negativ zu \\ bewerten sind.
}

K

Von Jürgen Meyerhoff und Ulrich Petschow

watt Leistung - tragen auf der ein MegaStärkung der regenerativen Energien bei und helfen so, Kohlendioxid-Emissionen zu vermeiden. In der Bundesrepublik gab es 1994 insgesamt 4.633 kleine Wasserkraftwerke mit einer Nettoerzeugung bzw. Einspeisung von 1,46 TWh Strom. Damit hatten sie an dem aus Wasserkraft gewonnenen Strom einen Anteil von 8,3 Prozent, bezogen auf den gesamten Stromverbrauch aus öffentlicher Versorgung einen Anteil von 0,33 Prozent. Geht man davon aus, daß bei der Erzeugung einer Kilowattstunde Strom in Deutschland durchschnittlich 0,57 Kilogramm Kohlendioxid entstehen, dann wurden durch die Stromerzeugung mittels kleiner Wasserkraftanlagen 826.500 Tonnen Kohlendioxid-Emissionen vermieden. Dies entspricht 0,09 Prozent der Gesamtemissionen. Abschätzungen des Potentials gehen davon aus, daß bis zum Jahr 2005 rund 1.000 kleine Wasserkraftanlagen mit einer durchschnittlichen Leistung von $70 \mathrm{~kW}$ zum bisherigen Bestand hinzukommen könnten. Dadurch würde die mögliche Menge eingesparter Kohlendioxid-Emissionen um 149.340 Tonnen erhöht.

Auf der anderen Seite ist die Errichtung oder Wiederinbetriebnahme der Anlagen aber of mit Beeinträchtigungen des Naturhaushaltes verbunden. Nachdem lange Zeit die biologische Gewässergüte im Mittelpunkt der umweltpolitischen Bemühungen stand, wurde in den letzten Jahren zunehmend die Bedeutung auch der Gewässerstrukturgüte hervorgehoben. Vor diesem Hintergrund können kleine Wasserkraftwerke einen Eingriff in die Fließgewässer darstellen, der zu einer Entfernung vom Leitbild des potentiell natürlichen Zustandes führt. Aktuell ist die Diskussion um das Für und Wider kleiner Wasserkraftwerke vor allem deshalb, weil
- bei einer großen Anzahl der betriebenen Anlagen die Wasserrechte auslaufen und die Frage der Neuvergabe ansteht,

- es - auch durch staatliche Förderung unterstiitzt - insbesondere in den neuen Bundesländern zu einer vermehrten Wiederinbetriebnahme von Altanlagen kommt und

- darüber hinaus an etlichen Standorten auch Neubauten geplant sind.

Für die Abwägung wurde vor allem die KostenNutzen-Analyse herangezogen: Ihr Vorteil ist, daß die Auswirkungen in monetären Größen gemessen und damit einheitlich bewertet werden. Weiterhin wurden die Bewertungsinstrumente Umweltverträglichkeitspriffung und ökobilanz einbezogen(1).

\section{Volkswirtschaftliche Abwägung}

Bei einer Betrachtung auf volkswirtschaftlicher Ebene sind zum einen die Kosten des anthropogenen Treibhauseffektes zu berücksichtigen. Sie dienen als Maßstab für die positiven Auswirkungen der Wasserkraftwerke. Befürworter stïtzen ihre Forderung nach finanzieller Unterstïtzung denn auch auf Berechnungen dieser Kosten, da der Gesellschaft im Vergleich zur Stromerzeugung mit kalorischen Kraftwerken, die fossile
Brennstoffe nutzen, ein Teil dieser Kosten erspart bleibt. Diese Vorteile würden Betreibern und Investoren von der Gesellschaft aber nicht abgegolten. Doch sind die bisher ermittelten Kostensätze (2) - seien es Vermeidungskosten oder Schadenskosten - mit großen Unsicherheiten behaftet, wobei die Angaben über Vermeidungskosten zur Zeit gesicherter sind.

Zum anderen müssen jedoch die Beeinträchtigungen des Naturhaushaltes einbezogen werden. Studien zur ökonomischen Bewertung von Natur und Landschaft zeigen, daß eine deutliche Nachfrage nach dem Schutz von Natur und Landschaft besteht. Die Höhe der Wertschätzung liegt zum Teil deutlich über den Kosten, die z. B. aus der Verhinderung des Artensterbens in Deutschland oder der Errichtung eines Nationalparks Donau-Auen entstehen würden. Allerdings haben bisherige Studien vor allem Programme zum Schutz von Arten oder zum Erhalt bestimmter Kulturlandschaften beurteilt. Es konnte jedoch u.a. auf die Ergebnisse einer monetären Bewertung von Biotopen zurückgegriffen werden. Sie gehen auf einen Bewertungsansatz zurïck, der im Zusammenhang mit der Eingriffsregelung des Bundesnaturschutzgesetzes entwickelt wurde (3).

Die Zusammenführung der positiven und negativen Effekte erfolgte in zwei Schritten: Zunächst wurden die Vorteile der Stromerzeugung ohne Kohlendioxid-Emissionen an Hand verschiedener Kostenansätze bewertet (vgl. Tabelle 1). Je nach Kostenansatz ergeben sich bei insgesamt 826.500 Tonnen vermiedenen Kohlendioxid-Emissionen positive externe Effekte in Höhe von 42 bis 601 Mio. DM. Zum Vergleich: Die externen Kosten der gesamten Kohlendioxid-Emissionen der Bundesrepublik liegen nach diesen Kostensätzen in einer

Tabelle 1: Vermiedene externe Kosten durch kleine Wasserkraftanlagen (Nicht-EVU) im Jahr 1994

\begin{tabular}{|c|c|c|c|c|c|c|}
\hline \multirow[t]{2}{*}{$\begin{array}{l}\text { Leistung } \\
\text { in } \mathrm{kW} \\
\end{array}$} & \multicolumn{2}{|c|}{$\begin{array}{l}\text { Vermiedene } \\
\mathrm{CO}_{2} \text {-Emissionen in } \uparrow\end{array}$} & \multicolumn{4}{|c|}{ Durchschnithlich vermiedene externe Kosten pro Jahr und Anlage } \\
\hline & Gesamt & $\begin{array}{l}\text { Durch- } \\
\text { schnitt } \\
\text { pro Anlage }\end{array}$ & $\begin{array}{l}\text { GEMIS } 3.0 \\
(1997) \\
50 \mathrm{DM} /+\mathrm{CO}_{2}\end{array}$ & $\begin{array}{l}\text { Fankhauser } \\
\text { (1995) } \\
115 \mathrm{DM} / \mathrm{C} \mathrm{CO}_{2}\end{array}$ & $\begin{array}{l}\text { INFRAS et al. } \\
\text { (1996) } \\
135 \mathrm{DM} / \mathrm{f} \mathrm{CO}_{2}\end{array}$ & $\begin{array}{l}\text { Hohmeyer, } \\
\text { Gärtner (1992) } \\
728 \mathrm{DM} / \mathrm{I} \mathrm{CO}\end{array}$ \\
\hline$<50$ & 120.384 & 41 & 2.000 & 4.715 & 5.535 & 29.848 \\
\hline $50-100$ & 82.308 & 145 & 7.250 & 16.675 & 19.575 & 105.560 \\
\hline $100-200$ & 105.165 & 314 & 15.700 & 36.110 & 42.390 & 228.592 \\
\hline 200-500 & 157.890 & 663 & 33.150 & 76.245 & 89.505 & 482.664 \\
\hline $500-1.000$ & 92.112 & 1.265 & 63.250 & 145.475 & 170.775 & 920.920 \\
\hline $\begin{array}{l}\text { Summe aller } \\
\text { Anlogen }\end{array}$ & 557.859 & & 27.892 .950 & 64.153 .785 & 75.310 .965 & 406.121 .352 \\
\hline
\end{tabular}


Tabelle 2: Durch positive externe Effekte kompensierbare Eingriffsfläche (in qm)

\begin{tabular}{|c|c|c|c|c|c|c|}
\hline $\begin{array}{l}\text { Biotop- } \\
\text { beweriung } \\
\mathrm{CO}_{2} \text {-Kosten }\end{array}$ & \multicolumn{2}{|c|}{$\begin{array}{l}\text { Fonds-Modell } \\
\text { 798,- DM/qm }\end{array}$} & \multicolumn{2}{|c|}{$\begin{array}{l}\text { Investitionsmodell } \\
\text { 448,- DM/qm }\end{array}$} & \multicolumn{2}{|c|}{$\begin{array}{l}\text { biotopspezifische } \\
\text { Entschädigungsforderung } \\
28,-D M / q m\end{array}$} \\
\hline Leistung in $\mathrm{kW}$ & $<50$ & $50 \cdot 100$ & $<50$ & $50 \cdot 100$ & $<50$ & $50 \cdot 100$ \\
\hline \multicolumn{7}{|c|}{ Quadratmeter kompensierbare Eingriffstläche } \\
\hline GEMIS 3.0 & 71 & 252 & 127 & 450 & 2.038 & 7.208 \\
\hline Fankhouser & 165 & 581 & 293 & 1.036 & 4.688 & 16.580 \\
\hline INFRAS & 193 & 682 & 343 & 1.216 & 5.503 & 19.463 \\
\hline $\begin{array}{l}\text { Hohmever/ } \\
\text { Görnner }\end{array}$ & 1.041 & 3.682 & 11.855 & 6.559 & 29.677 & 104.958 \\
\hline
\end{tabular}

Spannweite von 45 bis 653 Milliarden Mark Im zweiten Schritt wurden dann u.a. die Ansätze zur monetären Bewertung von Biotopen herangezogen. Je nach dort verwendetem Modell ergeben sich recht unterschiedliche Werte für die Ausgleichszahlung pro Quadratmeter Biotop (vgl. Tabelle 2). Aufbauend auf diesen Werten wurde dann berechnet, bis zu welchem Flächenumfang die kohlendioxidfreie Energieerzeugung einen Eingriff in ein naturnahes Fließgewässer ,,aufwiegen" würde. Danach könnte im für die Wasserkraft ,ungünstigsten“ Fall (hoher Wert der Biotope, geringe Vermeidungskosten für Kohlendioxid-Emissionen) bei Anlagen unter $50 \mathrm{~kW}$ Leistung nur ein Eingriff auf einer Fläche von 71 Quadratmetern kompensiert werden. Im ,günstigsten" Fall (geringe Entschädigungsforderung für Biotope, höchste Schadenskosten für Kohlendioxid-Emissionen) ergibt sich eine kompensierbare Fläche von ca. 30.000 Quadratmetern. Dies entspricht z. B. einem 50 Meter breiten und 600 Meter langen Streifen im Flußtal. Es ist davon auszugehen, daß eine Fläche dieser Größenordnung von der Stauhaltung oberhalb und der Erosionsstrecke unterhalb einer Wehranlage zumindest negativ beeinflußt würde.

Am Beispiel der Söse, einem Fließgewässer in Niedersachsen, wurde schließlich die Wirtschaftlichkeit eines Anlagenneubaus unter $50 \mathrm{~kW}$ Leistung näher betrachtet. Es zeigte sich, daß eine Anlage am beabsichtigten Standpunkt ohne öffentliche Förderung nicht wirtschaftlich wäre. Und nur wenn keine ökologischen Mindestanforderungen gestellt würden, wie dies von Interessenvertretern der Wasserkraftnutzung zum Teil gefordert wird, könnte die Anlage an die Schwelle zur Wirtschaftlichkeit heranrücken. Unterstellt werden dabei deutlich steigende Vermeidungskosten für Kohlendioxid-Emissionen. Werden aber ökologische Anforderungen an die Mindestwasserabflüsse oder die Durchgängigkeit des
Gewässers z.B. für Fische gestellt, dann ist diese Anlage bei Berücksichtigung der positiven und negativen Auswirkungen unwirtschaftlich.

Die Wirtschaftlichkeit hängt letztlich sehr stark vom jeweiligen Standort ab: Einerseits wird die Anlage umso wirtschaftlicher, je größer die mögliche Anlagenleistung am jeweiligen Standort ist. Auf der anderen Seite sind es aber oft die aus Sicht des Gewässerschutzes wichtigsten Gewässerabschnitte, die einen wirtschaftlichen Betrieb kleiner Wasserkraftwerke ermöglichen würden.

\section{- Fazit}

Auch wenn generelle Aussagen aufgrund der Abhängigkeit der Bewertung vom jeweiligen Standort nur schwer möglich sind, so konnte doch gezeigt werden, daß kleine Wasserkraftwerke aufgrund der Eingriffe in die Fließgewässer zu erheblichen Kosten führen. Es besteht eine deutliche Nachfrage nach dem Schutz von Natur und Landschaft, die auch von der Klimaschutzpolitik nicht vernachlässigt werden darf. Um einer Lösung des Konfliktes zwischen Klima- und Gewässerschutz näherzukommen, sollten daher folgende Schritte unternommen werden:

1. Zunächst sollten diejenigen Gewässer in der Bundesrepublik bestimmt werden, die aus Sicht des Gewässerschutzes in einem naturnahen Zustand erhalten oder wieder dem potentiell natïrlichen Zustand angenähert werden sollen.

2. Für diese Gewässer sind die Standorte mit ihren Potentialen zu ermitteln, an denen die Wasserkraft wirtschaftlich betrieben werden könnte. Dabei sind den Anlagen die positiven externen Effekte anzurechnen. Allerdings sollten für ihre Berechnung Vermeidungs- und nicht Schadenskosten herangezogen werden.

3. Sind Anlagen an diesen Standorten aus Sicht des Gewässerschutzes auch dann nicht akzeptabel, oder im Sinne von Punkt 2 nicht wirtschaftlich, wenn Ausgleichsmaßnahmen (Restwasser,
Zum Thema

Sechsmal im Jahr: Die Fachzeitschrift für Umweltrecht -

und damit sechsmal im Jahr ein kompletter Überblick über das Umweltrecht.

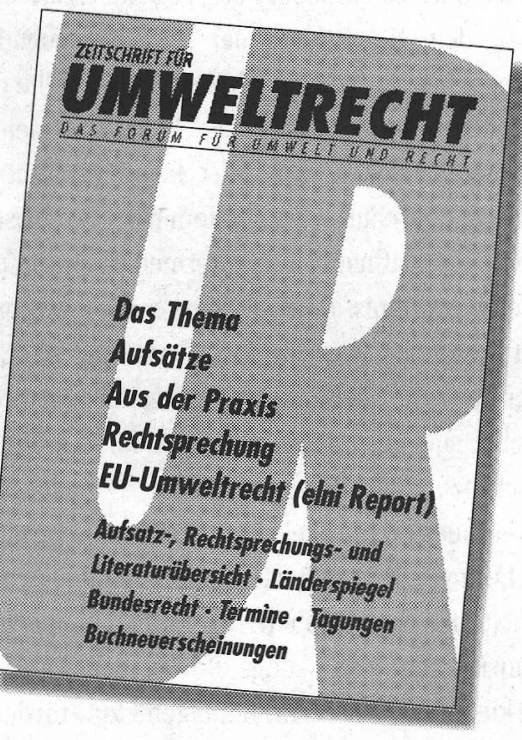

\section{Aktuelle wissenschaftliche Beiträge und Analysen}

- diskutieren Stand und Entwicklung des Umweltrechts.

\section{Ein umfangreicher Service-Teil}

- bringt die neveste Rechtsprechung,

- informiert über die aktuelle Gesetzesentwicklung auf Landes-, Bundes- und Europaebene und

- dokumentiert Aufsätze aus über einhundert Fachzeitschriften sowie wichtige Nachrichten, Termine und Buchneuerscheinungen.

Zu bestellen bei: Rhombos-Verlag

Kurfürstenstr. 17, 10785 Berlin

Tel. $030 / 2619461$, Fax 030/2616854 
Fischtreppen) durchgeführt würden, dann sind die entgangenen positiven Effekte aufgrund der Kohlendioxideinsparung dem Gewässerschutz als Kosten zuzuschreiben.

4. Diese Kosten infolge des Verzichts auf die Nutzung der Wasserkraft sollten mit in die Kosten für ein Programm zur Verbesserung des Gewässerschutzes aufgenommen werden. Sie wären dann der Nachfrage nach dem Schutz von Natur und Landschaft, die mit Hilfe der entsprechenden Methoden zu ermitteln wäre, gegenüberzustellen. Vor dem Hintergrund der heute bestehenden Knappheit an intakten Fließgewässern sollte der weiteren Erschließung des Potentials kleiner Wasserkraftwerke zunächst keine Priorität in der Klimaschutzpolitik zukommen. Die Kosten anderer Maßnahmen zur Vermeidung von Kohlendioxid-Emissionen sind bisher noch geringer, insbesondere wenn bedacht wird, daß die Eingriffe in die Fließgewässer in der Regel irreversibel sind. Erste Priorität sollte für die Klimaschutzpolitik dagegen haben, durch eine Veränderung der relativen Preise den Verursachern von Kohlendioxid-Emissionen die Knappheit der Athmosphäre als Aufnahmemedium anzuzeigen. Die heutige Praxis, den Bau von (kleinen) Wasserkraftanlagen zu fördern, gleichzeitig aber die kostenlose „Nutzung“ der Atmosphäre zu gestatten, führt bisher nur dazu, daß die Kohlendioxid-Emissionen weiter ansteigen und gleichzeitig schützenswerte Gewässer verbaut werden.

\section{Anmerkungen}

(1) Das Gutachten erscheint Ende Februar in der Reihe Texte des Umweltbundesamtes und kann über die Firma Werbung und Vertrieb, Ahornstr. 1-2, 10787 bezogen werden. Es wird außerdem als IÖW-Schriftenreihe 125/97 veröffentlicht.

(2) Herangezogen wurden die Ergebnisse folgender Studien: Fankhauser, S.: Valuing Climate Change. The Economics of the Greenhouse, London 1997; INFRAS/ ECONCEPT/ PROGNOS: Die vergessenen Milliarden. Externe Kosten im Energieund Verkehrsbereich, Bern 1996; Hohmeyer, 0., Gärnner, H.: The Costs of Climate Change. Report to the Commission of the European Communities, Karlsruhe 1992.

(3) Schweppe-Kraft, B.: Monetäre Bewertung von Biotopen und ihre Anwendung bei Eingriffen in Natur und Landschaft. Im Erscheinen.

\section{Die Autoren}

Jürgen Meyerhoff ist wiss. Mitarbeiter am Institut für Management in der Umveliplanung der TU Berlin, Ulrich Petschow ist wiss. Mitarbeiter am löW

Kontakt: Jürgen Meyerhoff, Tel. 030/314-73492, e-mail: Meyerhoff @imup.tu-berlin.de;

Ulrich Petschow, Tel. 030/8845940

Neuer Studienschwerpunkt an der FH Niederhein

\section{Fit für die ökologische Herausforderung}

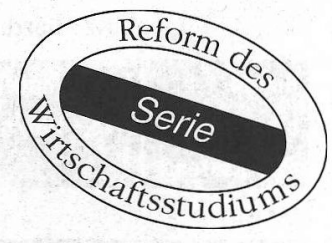

Betrieblicher Umweltschutz ist in der Praxis nach wie vor hauptsäichlich technischer Umweltschutz. Die zweite Folge der Hochschulserie stellt das neve Studienangebot "Ökonomie, Ökologie und Ethos" für Betriebswirte an der Fachhochschule Niederrhein vor, das die "betriebswirtschaftliche Lücke" schließen helfen soll.

S Von Martin Wenke eit dem Sommersemester 1997 gibt es fuir Studierende der Betriebswirtschaftslehre an der Fachhochschule Niederrhein in Mönchengladbach eine neue Alternative: Sie können fundierte Kenntnisse über Methoden des ÖkoControlling, organisatorische Konsequenzen der Einführung eines Umweltmanagementsystems und die Auswirkungen unterschiedlicher Instrumente der Umweltpolitik auf die Unternehmen erwerben.

Im Grundstudium umfaßt das neue Wahlfach vier Veranstaltungen $\mathrm{zu}$ den Grundlagen der Umweltökonomie sowie zu betriebs- und volkswirtschaftlichen Aspekten des Umweltschutzes (vgl. Kasten). Im Hauptstudium können die Studierenden dann im Rahmen eines Seminars ihre Kenntnisse in ausgewählten volks- oder betriebswirtschaftlichen Themenbereichen vertiefen. Hier kann es sowohl um die Zertifizierung eines betrieblichen Umweltmanagementsystems gemäß der EG-Öko-Audit-Verordnung, die Absatzchancen umweltfreundlicher Produkte oder die gesamt- oder regionalwirtschaftlichen Auswirkungen einer, ,ökologischen Steuerreform" gehen.

\section{Ausbildungsziel: Sensibilisierung}

Die Vermittlung umweltökonomischer Grundkenntnisse verfolgt das Ziel, die Studierenden mit Blick auf ihre zukünftige berufliche Tätigkeit für Umweltschutzbelange $\mathrm{zu}$ sensibilisieren. Hiermit verbunden ist die Hoffnung, daß sich z.B. der Mitarbeiter in der Einkaufsabteilung an die Veranstaltungen in der Hochschule erinnert und vorwiegend umweltfreundliche Vorprodukte, Halbzeuge oder Komponenten ordert, wovon er sich auch Kosteneinsparungen verspricht. Die in der Marketingabteilung beschäftigte FHN-
Absolventin könnte sich aufgrund ihrer Kenntnisse für die Entwicklung und den Vertrieb ökologischer Produktvarianten stark machen, da sie aufgrund entsprechender Untersuchungen von erheblichen Marktpotentialen ausgeht. Sie erwartet hierdurch jedoch auch einen Imagegewinn sowie eine Verbesserung der Marktstellung ihres Unternehmens.

\section{Zusatzkompetenz für} Absolventen und Hochschule

Die Entscheidung für das neue Studienangebot ermöglicht den Studierenden die Erlangung einer praxisrelevanten Zusatzkompetenz, die ihnen im immer härter werdenden Wettbewerb um qualifizierte Arbeitsplätze zugute kommt. Aufgrund ihrer Kenntnisse der ökonomisch-ökologischen Zusammenhänge werden sich die Absolventinnen und Absolventen außerdem auch als kompetente Gesprächspartner sowohl für die in vielen Unternehmen beschäftigten Umweltschutzbeauftragten aber auch für die mit dem technischen Umweltschutz betrauten Kolleginnen und Kollegen erweisen. Gerade mit Blick auf den betrieblichen Umweltschutz kommt der immer wieder geforderten Fähigkeit zur interdisziplinären Zusammenarbeit eine besondere Bedeutung zu. Diese Zusammenarbeit über die

\section{Reform des Wirtschaftsstudiums}

In der Hochschulserie "Reform des Wirtschaftsstudiums" werden in loser Folge interessante Studienangebote vorgestellt. Bisher ist in Ökologisch Wirtschoften 5/97 erschienen:

Reinhard Pfriem: Ökologische Ökonomie und Innovation kombinieren. Spezialisierung Diplom-Ökonomie mit ökologischem Schwerpunkt.

Weitere Beitrüge sind willkommen. 
(c) 20I0 Authors; licensee IÖW and oekom verlag. This is an article distributed under the terms of the Creative Commons Attribution Non-Commercial No Derivates License (http://creativecommons.org/licenses/by-nc-nd/3.o/), which permits unrestricted use, distribution, and reproduction in any medium, provided the original work is properly cited. 